\title{
Measurement of Rydberg positronium fluorescence lifetimes
}

\author{
A. Deller, A. M. Alonso, B. S. Cooper, S. D. Hogan, and D. B. Cassidy \\ Department of Physics and Astronomy, University College London, Gower Street, London, WC1E 6BT, United Kingdom
}

(Received 22 April 2016; published 29 June 2016)

\begin{abstract}
We report measurements of the fluorescence lifetimes of positronium (Ps) atoms with principal quantum numbers $n=10-19$. Ps atoms in Rydberg-Stark states were produced via a two-color two-step $1{ }^{3} S \rightarrow 2{ }^{3} P \rightarrow$ $n^{3} S / n^{3} D$ excitation scheme and subsequently detected after traveling $1.2 \mathrm{~m}$. The measured time-of-flight distributions were used to determine the mean lifetimes of the Rydberg levels, yielding values ranging from $3 \mu \mathrm{s}$ to $26 \mu \mathrm{s}$. Our data are in accord with the expected radiative lifetimes of Rydberg-Stark states of Ps.
\end{abstract}

DOI: 10.1103/PhysRevA.93.062513

\section{INTRODUCTION}

Studies involving positronium (Ps) atoms [1] cover a wide range of topics, including materials science [2], astrophysics [3], atomic and molecular scattering [4], tests of fundamental symmetries [5], and searches for new physics in the form of dark matter [6], extra dimensions [7], or forbidden decay modes [8]. Since Ps is composed only of leptons, it is amenable to (almost) exact QED calculations (e.g., $[9,10])$ and is in general an excellent system with which to test bound-state QED and recoil calculations [11]. To this end, precision measurements of several Ps properties have been undertaken, including the ground-state hyperfine splitting [12,13] and decay rates $[14,15]$, the $1^{3} S_{1}-2^{3} S_{1}$ interval [16], and the decay rate of the Ps negative ion $[17,18]$.

Any bound system comprising a particle-antiparticle pair is intrinsically unstable against annihilation. The groundstate annihilation lifetimes of Ps in vacuum are $125 \mathrm{ps}$ and $142 \mathrm{~ns}$ for the singlet and triplet states, respectively [1]. These lifetimes scale with $n^{3}$, but annihilation is strongly suppressed for states with $\ell \neq 0[19,20]$. Thus, in the absence of perturbing influences (such as collisions, electric fields, or radiation), Rydberg Ps lifetimes are determined primarily by their fluorescence lifetimes. These are dominated by radiative decay directly to the ground state but also contain contributions from all allowed radiative transitions to lower-lying states. Since the Ps reduced mass is $m_{e} / 2$, the radiative decay rates of Ps are approximately half those of the corresponding states in hydrogen [21].

Various experimental schemes could benefit significantly from the use of longer-lived excited-state Ps atoms in several ways: (1) Much longer flight paths provide extremely high resolution in time-of-flight (TOF) measurements [22], facilitate the application of Doppler correction methods [23], and allow for the separation of Ps production and interaction regions [24]. (2) The cross sections for Ps interactions with other systems can increase dramatically when Rydberg states are used [25,26], and choosing $n$ appropriately can act as a way to fine tune reaction dynamics [27]. (3) Long measurement times are necessary for high-resolution spectroscopy, which becomes feasible using Ps atoms in Rydberg states since annihilation is suppressed. As an example, microwave spectroscopy of transitions between Rydberg states with a resolution on the order of $\sim 100 \mathrm{kHz}$ can be achieved with measurement times of a few microseconds (e.g., [28]). (4) The large electric dipole moments of atoms in Rydberg states [29] can be used to control their translational motion via inhomogeneous electric fields [30,31]. This has been successfully demonstrated for many atomic and molecular Rydberg systems (e.g., [32-34]) and should be particularly useful for controlling Ps atoms, which are typically produced with large angular and velocity distributions (e.g., [35]).

Ps in well-defined Rydberg states can be produced using pulsed positron beams such that ground-state Ps atoms may be efficiently excited using intense pulsed laser radiation [23,36-38]. This has been done using the resonance-enhanced two-color two-photon $1{ }^{3} S \rightarrow 2^{3} P \rightarrow n^{3} S / n^{3} D$ excitation scheme first demonstrated by Ziock and co-workers [36]. Although this technique couples ground-state atoms to excited states with $s$ or $d$ character, the resulting Rydberg states will in general not themselves be pure $s$ or $d$ states.

In hydrogenic atoms, (e.g., H, D, or Ps) or for high$\ell$ (typically $\ell \geqslant 4$ ) states of nonhydrogenic species, the $\ell$ degeneracy in the absence of external fields effectively precludes photoexcitation of high Rydberg states described by a single value of $\ell$. Even the weakest stray electric fields break the spherical symmetry of the pure Coulomb potential, leading to mixing of the degenerate pure- $\ell$ states [39]. Under these circumstances it is more convenient to solve the Schrödinger equation in parabolic coordinates rather than spherical polar coordinates. The resulting eigenstates are then labeled according to their parabolic quantum numbers $n_{1}$ and $n_{2}$, and Stark states may be characterized using the index $k=n_{1}-n_{2}$ [29].

Atomic beam experiments have been reported in which stray electric fields were canceled to $\pm 20 \mu \mathrm{V} / \mathrm{cm}$ [28]. However, in experiments in which Ps is produced by the implantation of magnetically guided positrons into porous silica, canceling stray electric fields to this level is practically impossible, and motionally induced electric fields cannot be avoided [40]. Consequently, it is necessary to consider each excited state to possess mixed- $\ell$ character when determining fluorescence lifetimes, or blackbody-radiation-induced decay rates, even for photoexcitation in spatial regions with nominally zero electric fields. The spectral intensities of the transitions to these states from the ground, or a low-lying intermediate state, are then governed by the amount of low- $\ell$ character (i.e., $s$ or $d$ character) they possess.

Here we present measurements of lifetimes of Ps atoms in Rydberg states with principal quantum numbers ranging from $n=10-19$. Many measurements of the fluorescence lifetimes of short-lived, low-angular-momentum Rydberg states of 
nonhydrogenic atoms have been performed in beams, and in laser-cooled ensembles (e.g., [41-44]). However, the long lifetimes of $\ell$-mixed hydrogenic Rydberg states make it difficult to achieve sufficiently long observation times in beam experiments to enable precise measurements. The development of Rydberg-Stark deceleration techniques and electrostatic trapping of samples initially traveling in beams [33,45,46] have permitted measurements of decay processes involving these states on time scales extending up to several milliseconds in room temperature and cryogenic environments $[47,48]$.

Some of the problems associated with measuring long Rydberg-state lifetimes are exacerbated in the case of Ps. In particular, typical Ps speeds are of the order of $10^{5} \mathrm{~ms}^{-1}$ [49-51], and so long flight paths are required in order to measure lifetimes on the microsecond time scale. The first attempt to directly measure Rydberg Ps fluorescence lifetimes was made by Jones et al. [23]. In this experiment $\sim 0.6-\mathrm{eV}$ Ps atoms were detected after flying for $0.5 \mathrm{~m}$ in just under $2 \mu \mathrm{s}$. No difference was observed in the TOF distributions for atoms in states with $n=15$ and $n=20$, which was attributed to both states having lifetimes much longer than the flight time as a result of $\ell$ mixing. Using a longer flight path $(1.2 \mathrm{~m})$, slower Ps atoms $(\sim 0.15 \mathrm{eV})$, and a wider range of $n$ states, we have observed fluorescence decay of Rydberg Ps. Our measurements are consistent with those of Jones et al. and yield lifetimes from 3 to $26 \mu \mathrm{s}$, in accord with the expected fluorescence lifetimes of Rydberg-Stark states.

\section{EXPERIMENTAL METHODS}

The experimental apparatus and techniques used to produce and detect Rydberg Ps atoms are described in detail elsewhere [52]. Low-energy positrons are obtained from a radioactive ${ }^{22} \mathrm{Na}$ source [53] and captured in a Surko-type buffer gas trap [54]. Approximately $10^{5}$ positrons are ejected from the trap every second and delivered to the target in a pulse with a time width of $\Delta t \approx 4 \mathrm{~ns}$ [55] and a Gaussian spatial profile with a FWHM of $3 \mathrm{~mm}$. The positron implantation energy is controlled by biasing the target electrode, shown schematically in Fig. 1.

The positronium formation target used was a thin mesoporous silica film, spin coated on a silicon substrate [56,57]. The target was made using the sol-gel method with TEOS (tetraethylorthosilicate) as a precursor to silicon dioxide, with a triblock copolymer Pluronic F-127 (poloxamer 407) [58]. After positron implantation, Ps is formed in the bulk material and then diffuses through the interconnected pore network. The emission time (1-10 ns [35]) and Ps energy (0.03$1 \mathrm{eV})[59,60]$ depend on how far the Ps travels through the target and hence on the positron beam implantation energy. The Ps production efficiency is approximately $0.25 / \mathrm{e}^{+}$, resulting in a dilute Ps gas in vacuum with an initial density of $\sim 10^{7} \mathrm{~cm}^{-3}$.

Two Nd:YAG pumped dye lasers (6 ns FWHM) were used to excite Ps atoms to Rydberg states in a resonance-enhanced two-color two-photon process. $1{ }^{3} S \rightarrow 2{ }^{3} P$ transitions were driven using an ultraviolet (UV) laser (Fluence $\approx 1 \mathrm{~mJ} / \mathrm{cm}$, $\Delta v=85 \mathrm{GHz}, \lambda=243.0 \mathrm{~nm})$, and $2{ }^{3} \mathrm{P} \rightarrow n^{3} \mathrm{~S} / n^{3} \mathrm{D}$ transitions were driven using an infra-red (IR) laser (Fluence $\approx 12 \mathrm{~mJ} / \mathrm{cm}, \Delta v=5 \mathrm{GHz}, \lambda=728-760 \mathrm{~nm})$. Both laser beams had large spot sizes $\left(\sim 0.5 \mathrm{~cm}^{2}\right)$ to ensure complete
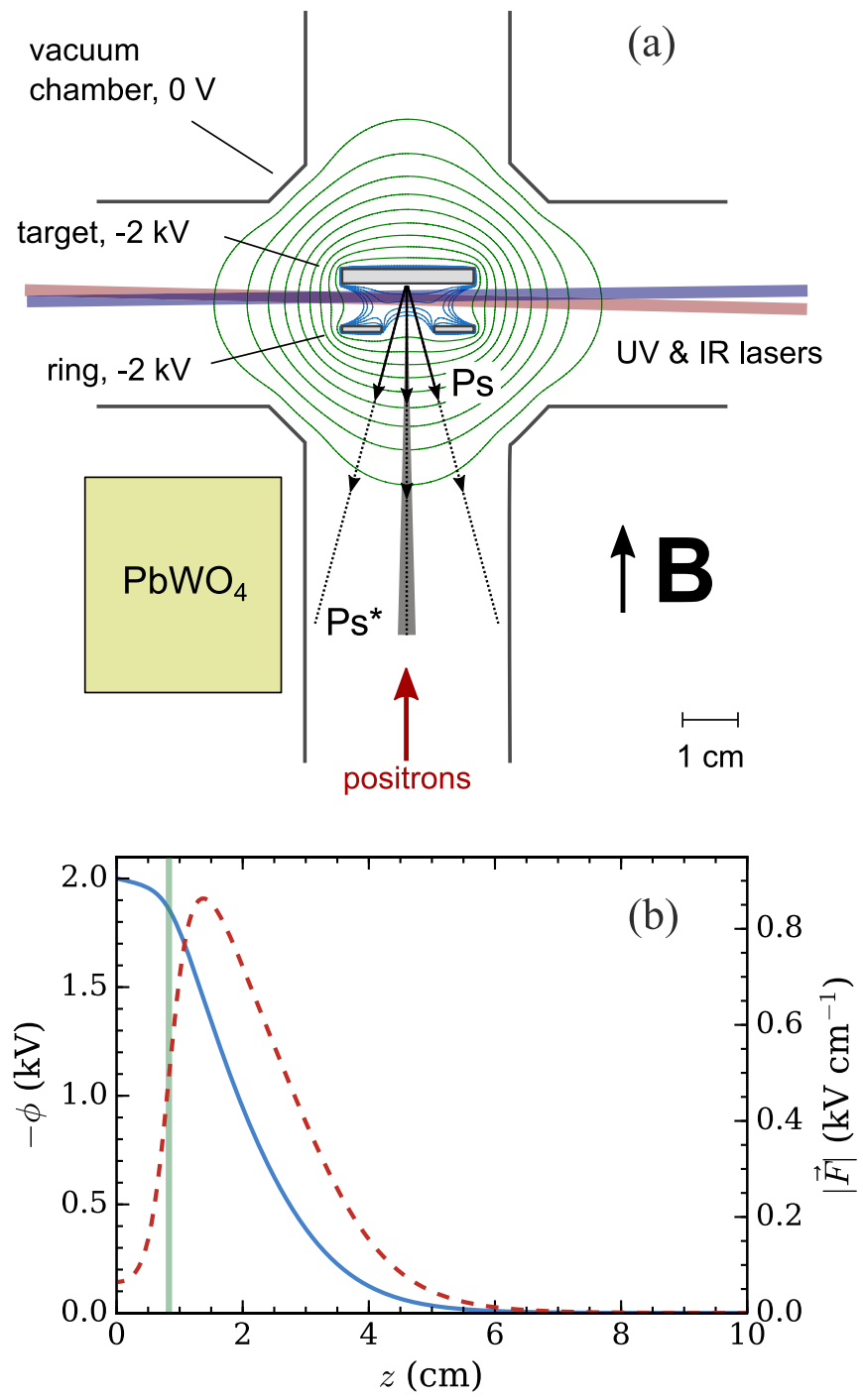

FIG. 1. (a) Schematic layout of the target chamber, indicating the Ps formation and excitation regions. The green lines of equipotential, calculated with a potential of $-2 \mathrm{kV}$ applied to the target and ring electrodes, range from -200 to $-1800 \mathrm{~V}$ in steps of $200 \mathrm{~V}$. The blue contours in the excitation region range from $1950 \mathrm{~V}$ to $1990 \mathrm{~V}$ in steps of $10 \mathrm{~V}$. The gray cone marks the range of emission angles that can subsequently be detected by a NaI detector located $1.2 \mathrm{~m}$ downstream. (b) The potential (solid line) and electric field (dashed line) along the beam axis. The vertical line indicates the position of the ring electrode.

spatial overlap with the Ps cloud, which at the time of excitation extends out from the target by less than $0.2 \mathrm{~cm}$. A large UV bandwidth was required to obtain a significant spectral overlap with the Doppler-broadened $1{ }^{3} S \rightarrow 2{ }^{3} P$ transition. This overlap is the main limitation on the overall efficiency for excitation of Rydberg states, and in the present experiments we estimate that we generate on the order of $10^{3}$ Rydberg atoms per pulse.

The silica film was mounted on a planar (target) electrode, used to control the positron implantation energy. A second (ring) electrode was mounted parallel to the target, with an offset of $7.8 \mathrm{~mm}$ and a 10 -mm-diameter entrance hole. This arrangement is used to control the electric field in the Ps 


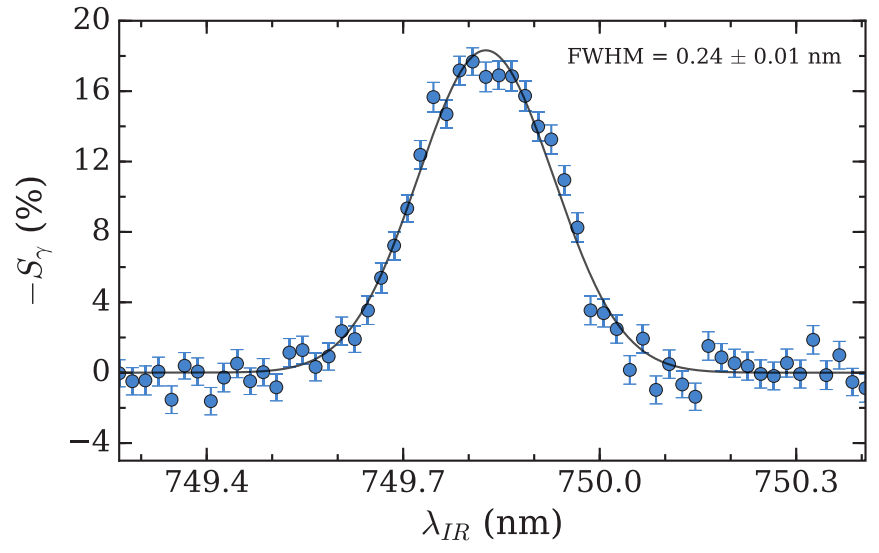

FIG. 2. Spectrum of the Doppler-broadened $2{ }^{3} \mathrm{P} \rightarrow 12{ }^{3} \mathrm{~S} / 12{ }^{3} \mathrm{D}$ transition measured using SSPALS. The solid line is a Gaussian fit to the data and yields a width of $0.24 \mathrm{~nm}$ FWHM, corresponding to $\sim 130 \mathrm{GHz}$.

excitation region without changing the positron implantation energy. Both electrodes were biased to $-2 \mathrm{kV}$ in order to eliminate the electric field in the excitation region. However, because of the large entrance aperture the resulting electric field was $\sim 100 \mathrm{~V} \mathrm{~cm}^{-1}$ (see Fig. 1).

The production and photoexcitation of Ps atoms was monitored via their annihilation radiation using a technique known as single-shot positron annihilation lifetime spectroscopy (SSPALS) [61]. This method uses a fast $\gamma$-ray detector [62] connected to an oscilloscope to monitor the time dependence of Ps annihilation radiation. Since Rydberg Ps atoms live for much longer than ground-state Ps atoms, they can be detected via the presence of a delayed annihilation signal [52]. In the present experiment a signal is obtained from atoms that collide with the chamber walls around $500 \mathrm{~ns}$ after they have been excited.

We can in general observe laser-induced transitions that affect Ps decay rates by measuring changes in the timedependent $\gamma$-ray signal [52]. This leads to a signal parameter $S_{\gamma}$ that can be used to characterize Ps-laser interactions. Figure 2 shows the spectrum of the $2{ }^{3} \mathrm{P} \rightarrow 12{ }^{3} \mathrm{~S} / 12^{3} \mathrm{D}$ transition measured in this way. The $\sim 130 \mathrm{GHz}$ width of this transition is consistent with Doppler broadening for Ps with a transverse speed of $\sim 10^{5} \mathrm{~ms}^{-1}$, and the bandwidth of the excitation lasers. The SSPALS methodology is not well suited for studying very long-lived Ps atoms, since they may travel a long distance from the excitation region and leave the detector field of view. In order to detect these atoms, a NaI scintillator coupled to a photomultiplier tube was used, as indicated in Fig. 3.

In the experiments the incident positron beam was magnetically steered through a $45^{\circ}$ angle with respect to the trap axis before reaching the target. This provided an excitation region in which the electric and magnetic fields could be aligned and still allow Rydberg Ps emitted normal to the silica film to travel along a drift tube rather than towards the positron trap (see Fig. 3). The NaI detector was located $1.2 \mathrm{~m}$ from the production region, and the vacuum chambers collimated the Ps such that only atoms emitted with angles less than $1.5^{\circ}$ would be transmitted to the detection region (see Fig. 3). By counting the arrival time of $\gamma$ rays registered at the end of the flight path

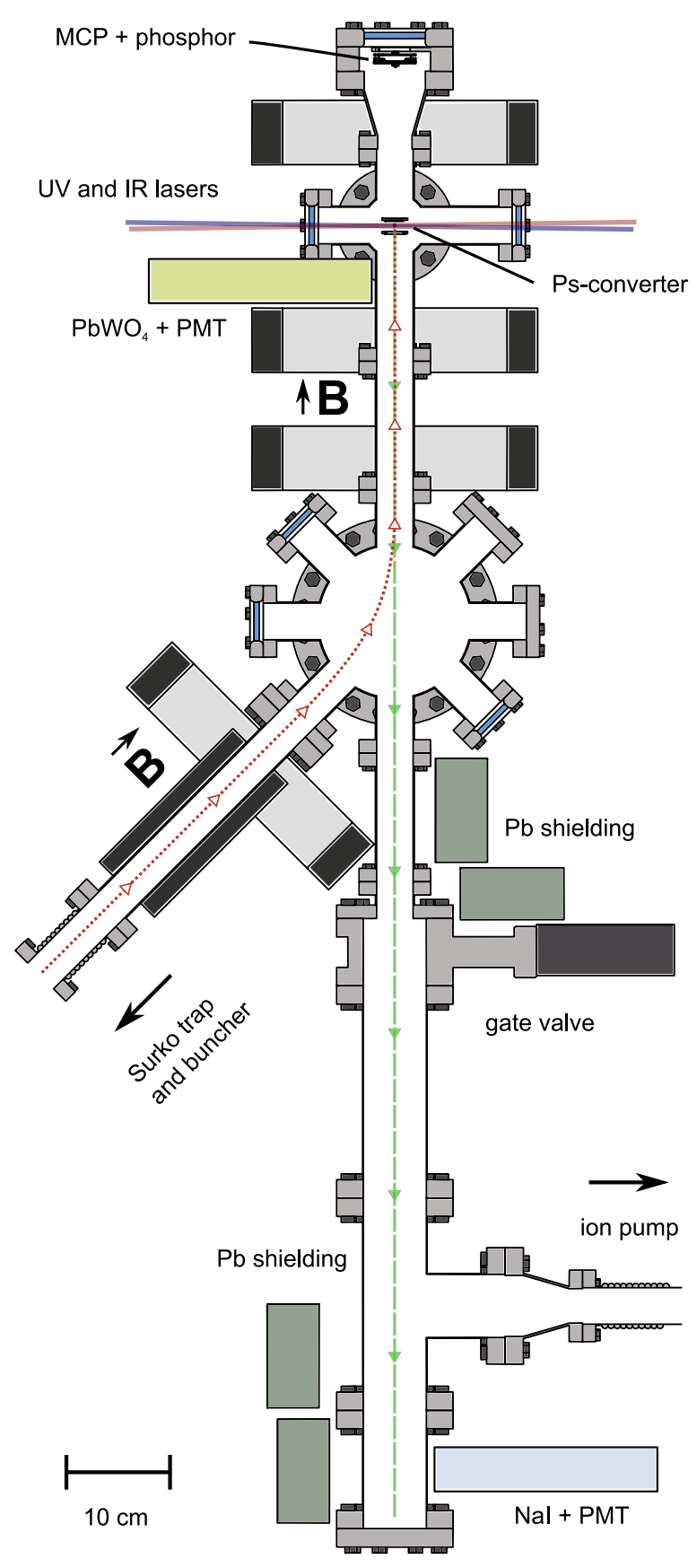

FIG. 3. Rydberg Ps time-of-flight setup. Positrons from a trap (red dots) are guided along the magnetic field lines of the solenoid and four coils (black) through an angle of $45^{\circ}$ and implanted into a mesoporous silica target. Ps atoms emitted almost normal to the film and excited to sufficiently long-lived states travel $1.2 \mathrm{~m}$ along the straight flight path (green dashes) to annihilate near the $\mathrm{NaI} \gamma$-ray detector.

relative to the positron implantation time, TOF spectra were obtained.

Two detectors were used to perform the TOF measurements, one with a $76 \mathrm{~mm}$ diameter $\mathrm{NaI}$ scintillator and one with a $50 \mathrm{~mm}$ diameter scintillator. The data from both detectors were combined. $\mathrm{NaI}$ has a scintillation decay constant of $0.23 \mu \mathrm{s}$, which is not appropriate for SSPALS but is suitable for single event counting at a low rate. The NaI detector output was 


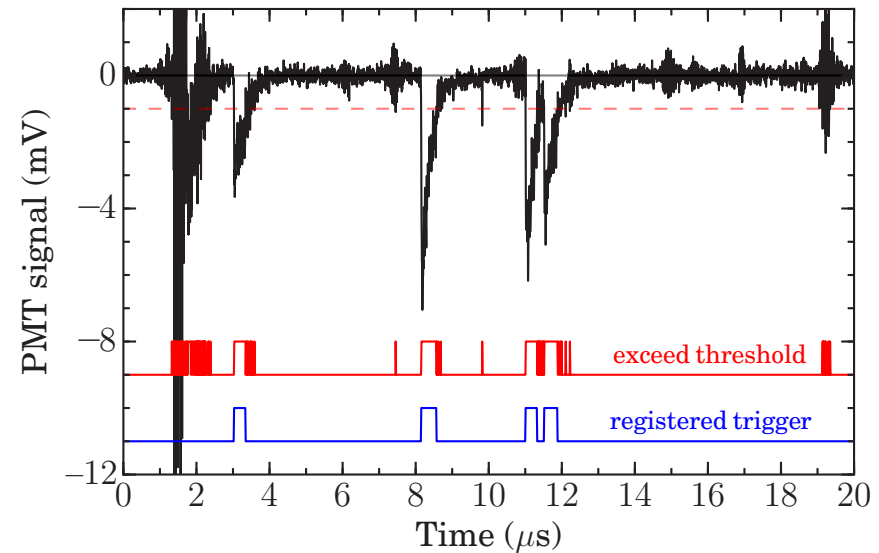

FIG. 4. The photomultiplier tube signal recorded for a single Rydberg-Ps-formation cycle (black). The sections where the signal exceeds a threshold of $-1 \mathrm{mV}$ (dashed line) are shown in red. The leading edges of above-threshold sections that are at least $100 \mathrm{~ns}$ wide are registered as trigger events (blue).

recorded with an oscilloscope, triggered by the trap dump sequence. The wave forms were recorded in 6-ns time bins for a period of $20 \mu \mathrm{s}$.

The occurrence of trigger events was determined by postprocessing the recorded $\mathrm{NaI}$ wave forms; a trigger was registered if the signal fell below a threshold of $-1 \mathrm{mV}$ for a period of at least $100 \mathrm{~ns}$. The time of the leading edge, the amplitude, and the width of each trigger event were recorded (see Fig. 4). The timing resolution was determined primarily by the oscilloscope sample interval $(\sim 6 \mathrm{~ns})$ rather than the long decay time of the NaI. As the wave form was recorded in its entirety, the threshold conditions could be adjusted after the fact to minimize true event rejection and reduce the rate for double counting or false triggers. The absolute detection rate was between 1 and 4 events per shot, and trigger pileup was therefore negligible.

The registered events were collected in a histogram comprising 500-ns time bins to generate the corresponding TOF spectra. Figure 5 shows a TOF spectrum obtained with the IR laser tuned to excite Rydberg states with $n=18$, along with a background measurement with the IR laser off-resonance. The difference between the two spectra corresponds to the signal from Rydberg Ps atoms. For these data, the total signal event rate is $\sim 0.5 \mathrm{~Hz}$. The decaying background is assumed to come from the annihilation of positrons that are backscattered from the target or have otherwise become temporarily trapped in the beamline. An exponential fit to the background yields a decay rate of $0.33 \mu \mathrm{s}^{-1}$.

\section{EXCITED-STATE FLUORESCENCE LIFETIMES}

The fluorescence lifetime, $\tau_{n \ell}$, of a pure- $\ell$ Rydberg state is given by the inverse of the sum over the Einstein $A$ coefficients associated with all allowed decay pathways to lower-lying states $\left|n^{\prime} \ell^{\prime}\right\rangle$, i.e.,

$$
\begin{aligned}
\tau_{n \ell} & =\Gamma_{n \ell}^{-1} \\
& =\left[\sum_{n^{\prime} \ell^{\prime}} A_{n^{\prime} \ell^{\prime}, n \ell}\right]^{-1},
\end{aligned}
$$

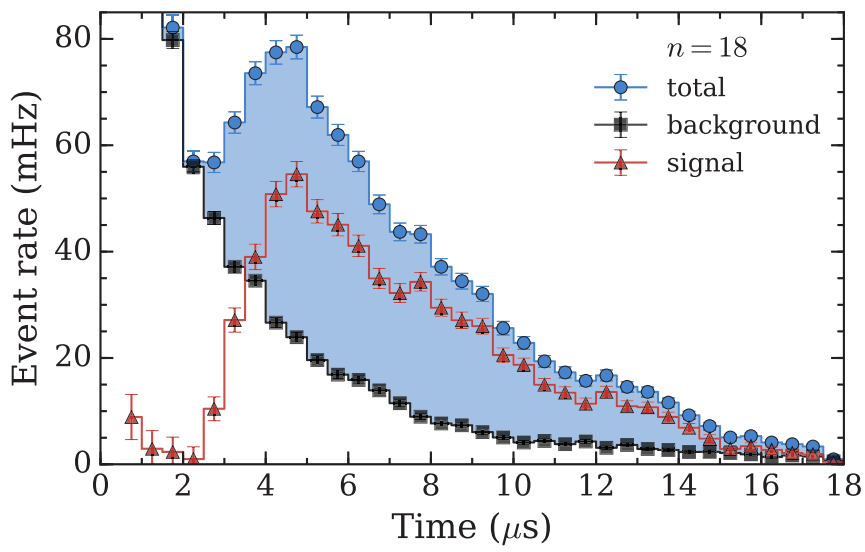

FIG. 5. Histogram of the event detection rate per 500-ns time bin, with the IR laser tuned to excite Ps atoms to states with $n=18$, or off resonance, as indicated in the legend. The background-subtracted histogram (i.e., total minus background) constitutes a Rydberg Ps TOF spectrum (signal).

where [29]

$$
A_{n^{\prime} \ell^{\prime}, n \ell}=\frac{2 e^{2} \omega_{n^{\prime} \ell^{\prime}, n \ell}^{3}}{3 \epsilon_{0} h c^{3}} \frac{\ell_{\max }}{2 \ell+1}\left|\left\langle n^{\prime} \ell^{\prime}|r| n \ell\right\rangle\right|^{2},
$$

and $\omega_{n^{\prime} \ell^{\prime}, n \ell}=2 \pi v_{n^{\prime} \ell^{\prime}, n \ell}$ is the angular frequency corresponding to the energy difference between the states, $\ell_{\max }=$ $\max \left(\ell, \ell^{\prime}\right)$, and $e, \epsilon_{0}, h$, and $c$ are the electron charge, the vacuum permittivity, the Planck constant, and the speed of light in vacuum, respectively. The corresponding fluorescence lifetimes of the $s, p, d$, and "circular" $(\ell=n-1)$ states of Ps with values of $n$ in the range from 10 to 20 are displayed in Fig. 6.

When the Rydberg states are photoexcited in a stray or weak background electric field, as in the experiments described here, the mixed- $\ell$ character of the resulting states leads to fluorescence lifetimes that lie between those of the short-lived

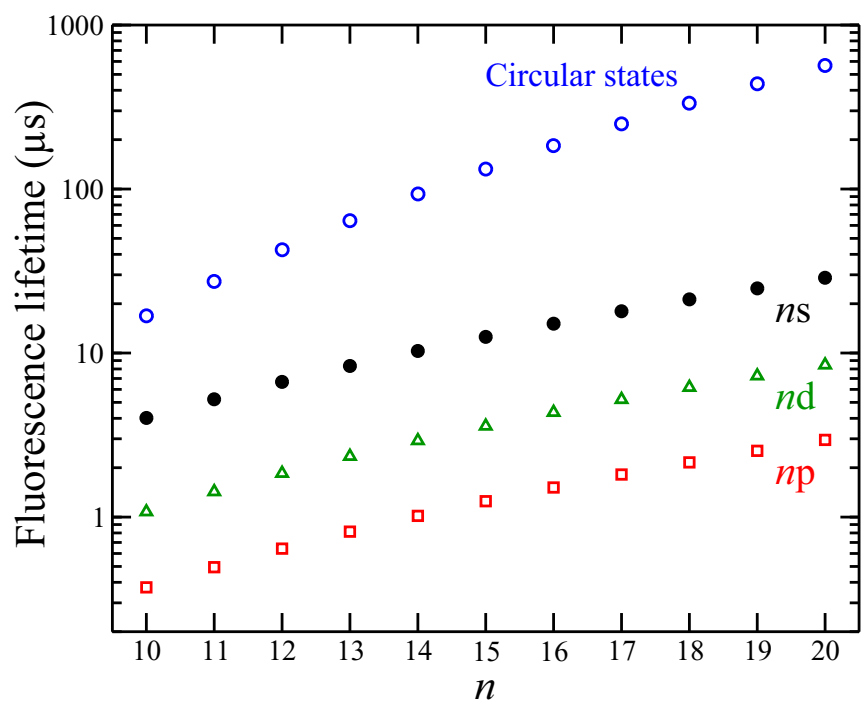

FIG. 6. Fluorescence lifetimes of pure $n s, n p, n d$, and circular $\ell=n-1$ Rydberg states of Ps with values of $n$ ranging from 10 to 20 . 
low- $\ell$ states and the long-lived, high- $\ell$ circular states (see Fig. 6). The fluorescence rate, $\Gamma_{n n_{1} n_{2} m}$, of each $\ell$-mixed $\left|n n_{1} n_{2} m\right\rangle$ state can be determined by summing over the decay rates of the $|n \ell\rangle$ components into which it can be transformed, weighted by the squares of the transformation coefficients, $\left\langle n n_{1} n_{2} m \mid n \ell m\right\rangle$, where [29]

$$
\begin{aligned}
\left\langle n n_{1} n_{2} m \mid n \ell m\right\rangle= & (-1)^{\left[\left(1-n+m+n_{1}-n_{2}\right) / 2\right]+\ell} \sqrt{2 \ell+1} \\
& \times\left(\begin{array}{ccc}
\frac{n-1}{2} & \frac{n-1}{2} & \ell \\
\frac{m+n_{1}-n_{2}}{2} & \frac{m-n_{1}+n_{2}}{2} & -m
\end{array}\right),
\end{aligned}
$$

with the last term in brackets representing a Wigner-3J symbol. Therefore the fluorescence lifetime, $\tau_{n n_{1} n_{2} m}$, is [63]

$$
\begin{aligned}
\tau_{n n_{1} n_{2} m} & =\Gamma_{n n_{1} n_{2} m}^{-1} \\
& =\left[\sum_{n \ell}\left|\left\langle n n_{1} n_{2} m \mid n \ell m\right\rangle\right|^{2} \Gamma_{n \ell}\right]^{-1} .
\end{aligned}
$$

The dependence of these fluorescence lifetimes on the differences between the parabolic quantum numbers, $k=n_{1}-n_{2}$, for excited states of Ps with $n=10$ and 20, and $|m|=0,1$, and 2 are displayed in Fig. 7. These fluorescence lifetimes exhibit a strong dependence on the value of $|m|$. In hydrogenic atoms, the pure $n s$ excited states live longer than the $n p$ states because they cannot decay directly to the $1 s$ ground state (see Fig. 6). The $\ell$-mixed Rydberg states with $m=0$ all possess equal amounts of "long-lived" $s$ character [see Eq. (4)]. However, they possess significantly different amounts of "shorter-lived" $p$ character. Because of their approximately symmetric electron charge distributions, and therefore electric dipole moments close to zero, states with low values of $|k|=$ $\left|n_{1}-n_{2}\right|$ possess little $m=0 p$-character, while states with high values of $|k|$ which exhibit asymmetric electron charge distributions and nonzero electric dipole moments possess significant $m=0 p$-character. Consequently, for the high- $|k|$

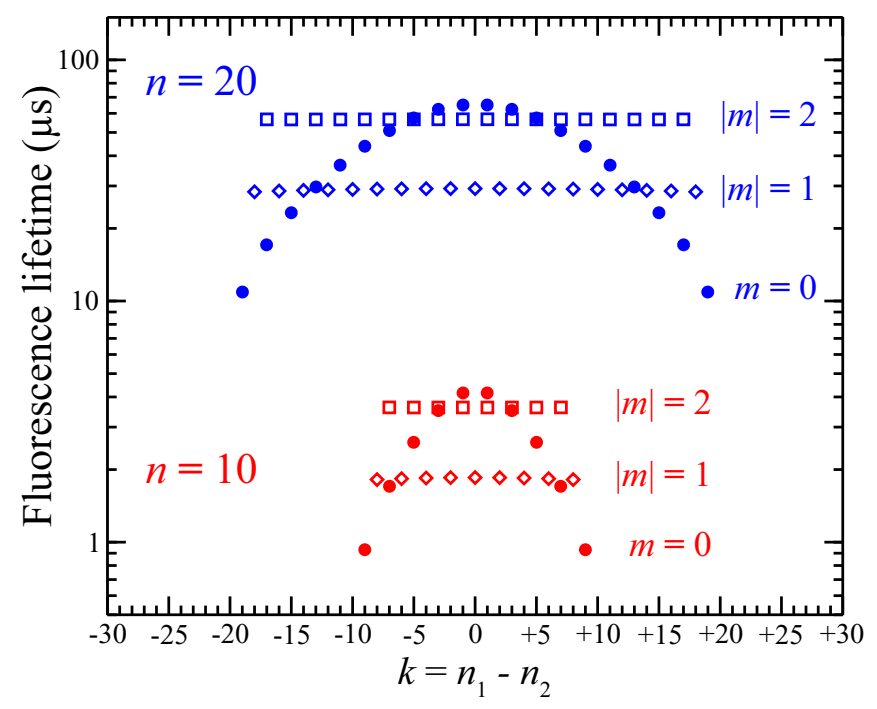

FIG. 7. Fluorescence lifetimes of $\ell$-mixed $\left|n n_{1} n_{2} m\right\rangle$ Rydberg states of Ps with $n=10$ and $n=20$, and $|m|=0,1$, and 2. Each state is denoted by the difference between the parabolic quantum numbers $k=n_{1}-n_{2}$.

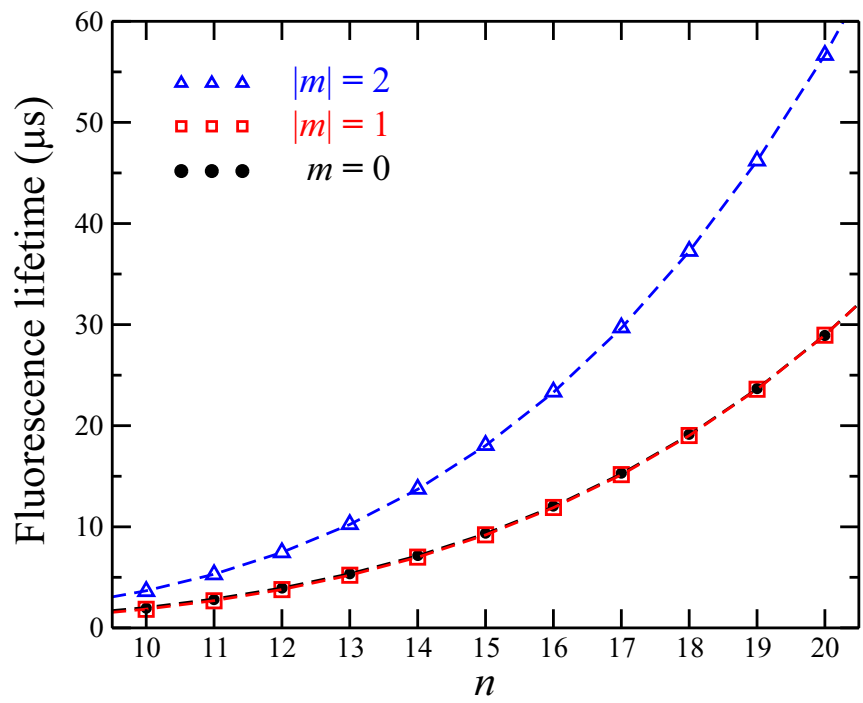

FIG. 8. Average fluorescence lifetimes of $\ell$-mixed $\left|n n_{1} n_{2} m\right\rangle$ Rydberg states of Ps for which $m=0,|m|=1$ and $|m|=2$.

states the contributions of the "longer-lived" $s$ components to the total fluorescence lifetimes are canceled by their "short-lived" $p$ character, while this is not the case for the low- $|k|$ states. This is the origin of the strong dependence of the $m=0$ fluorescence lifetimes on the value of $k$. For higher values of $|m|$ the contributions to the $\ell$-mixed Rydberg states from each value of $\ell$ balances, and because the fluorescence lifetimes increase monotonically with increasing values of $\ell$, a corresponding increase in the fluorescence lifetimes of the mixed states is seen as $|m|$ increases.

In the experiments reported here the Rydberg state photoexcitation process is in general saturated. Therefore for each value of $|m|$ the observed fluorescence lifetime is expected to be approximately equal to the mean lifetime across each $\left|n n_{1} n_{2} m\right\rangle$ manifold. The dependence of these mean lifetimes on the value of $n$ can be seen in Fig. 8. For each value of $|m|$ considered, the lifetimes $\tau_{\mathrm{fl},|m|}$ scale with $n^{4}$ (dashed curves):

$$
\begin{aligned}
\tau_{\mathrm{fl}, 0} & \simeq 1.8 \times 10^{-4} n^{4} \mu \mathrm{s} \\
\tau_{\mathrm{fl}, 1} & \simeq 1.8 \times 10^{-4} n^{4} \mu \mathrm{s} \\
\tau_{\mathrm{fl}, 2} & \simeq 3.5 \times 10^{-4} n^{4} \mu \mathrm{s} .
\end{aligned}
$$

\section{RESULTS AND DISCUSSION}

The experiments reported here were carried out using Ps atoms excited to Rydberg states, with TOF spectra obtained for atoms with values of $n$ in the range 10-20. Figure 9(a) shows spectra recorded using SSPALS by scanning the IR laser wavelength and driving $2{ }^{3} P \rightarrow n{ }^{3} S / n^{3} D$ transitions for $n \geqslant$ 10 . For $n \geqslant 23$, separate $n$ states can no longer be resolved, owing to Doppler and Stark broadening, and the bandwidth of the excitation lasers.

The inversion in the sign of the signal parameter $S_{\gamma}$ for $n \geqslant 21$ is related to whether or not atoms are able to leave the excitation region without field ionization taking place. The data are analyzed such that $S_{\gamma}$ is negative when Ps annihilation 


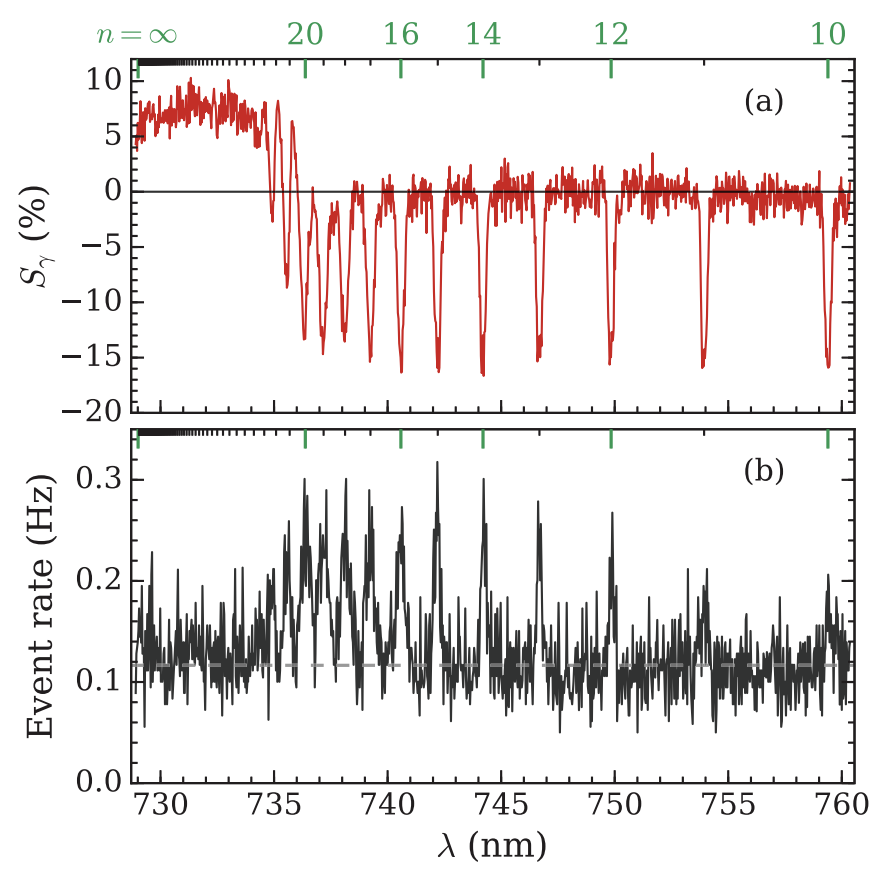

FIG. 9. (a) SSPALS signal as the wavelength of the IR laser is tuned over the range of $n=10-\infty$. The expected position of each $n$ state is indicated along the top axis. (b) The event rate registered by the $\mathrm{NaI}$ detector positioned $1.2 \mathrm{~m}$ from the Ps converter over the time window from $0.8-18 \mu$ s after positron implantation. Each data point represents 300 trap cycles.

is delayed relative to the 142-ns ground-state lifetime [52]. However, if atoms annihilate on a shorter time scale (e.g., following ionization), then $S_{\gamma}$ will be positive.

The magnitude of the largest electric fields experienced by the Ps is $\sim 0.9 \mathrm{kV} \mathrm{cm}^{-1}$ [see Fig. 1(b)]. This allows complete extraction of Ps up to $n \sim 19$; for higher $n$ states field ionization begins to filter different Stark states, until eventually no atoms are able to leave the excitation region. The data in Fig. 9(a) therefore indicate that all states with $n<20$ are able to leave the interaction region but atoms with values of $n \geqslant 22$ cannot. In the intermediate region some Stark states are transmitted while others are ionized [38]. This is visible also in the data presented in Fig. 9(b), which shows the NaI signal measured in the same scan and indicates that negative $S_{\gamma}$ measurements of Fig. 9(a) are correlated with the ability of Rydberg atoms to reach the $\mathrm{NaI}$ detector.

Positronium atoms created in mesoporous silica films [57] are emitted into the internal voids with an initial energy of $\sim 1 \mathrm{eV}$ [64], which decreases rapidly following collisions with the internal surfaces [65]. Increasing the positron beam implantation energy, and hence depth [66], increases the number of Ps-surface interactions and results in the emission of colder Ps [59]. The $\sim 5-\mathrm{nm}$ pores in the films used in the present work are such that the minimum Ps emission energy is determined by quantum confinement, with a void zero-point energy of $E_{0} \sim 30 \mathrm{meV}$ [60]. The Ps energy can therefore vary from $0.03 \mathrm{eV}$ to $1 \mathrm{eV}$, and the expected flight times to the $\mathrm{NaI}$ detector range from 3 to $16 \mu \mathrm{s}$.

For the TOF measurements shown here the positron beam implantation energy was $2 \mathrm{keV}$, which results in a mean longitudinal Ps emission energy of the order of $150 \mathrm{meV}$ [35].
The distribution of Rydberg Ps atoms will be modified because the spectral, spatial, and temporal profiles of the excitation lasers select a subset of the ground-state atoms [27]. Large laser beam sizes ( $0.5 \mathrm{~cm}$ FWHM) were used to maximize the number of Rydberg atoms produced, and to minimize the extent to which shot-to-shot variations or beam drifting could affect the Rydberg Ps velocity distributions.

A series of TOF measurements were made for Rydberg Ps atoms excited to states with values of $n$ ranging from 10 to 20 . Data were recorded for $60 \mathrm{~s}$ at each $n$, and two background spectra were recorded with the IR laser off resonance $(\lambda=$ $751.465 \mathrm{~nm}$ ). This was repeated for a total of $65 \mathrm{~h}$, during which the order of measurements for each 13-min series was randomly shuffled. The corresponding background-subtracted event rates per 500-ns time bin are shown in Fig. 10 for the even $n$ scans. It is evident from the data that more events are detected at later times $(t>8 \mu \mathrm{s})$ for the higher- $n$ states, as expected. The maximum detection rate occurs at $t \sim 5 \mu \mathrm{s}$ and is similar in amplitude for all spectra with $n \geqslant 13$. The similarity of these spectra indicates that the lifetimes of the corresponding Rydberg states are at least of the same order as the flight times (i.e., 3-18 $\mu$ s), which is consistent with the fluorescence lifetimes calculated in Sec. III.

The main factors that determine the shape of the TOF spectra and total event rate are the underlying (laser-selected) Ps velocity distribution, and any loss mechanisms. The former depends on the details of the Ps production and emission from the silica sample and is expected to be the same for all TOF measurements. The latter includes any processes that might prevent Rydberg Ps atoms from reaching the NaI detector.

The low density of Ps atoms produced in the experiment $\left(\sim 10^{7} \mathrm{~cm}^{-3}\right)$ means that collisional losses are not expected. Similarly, secondary electrons produced by the incident positron beam will not be present at the time of Ps excitation and therefore are also not expected to affect the Rydberg Ps atoms. The inhomogeneous electric field outside the Ps excitation region could slightly alter the trajectories of Rydberg Ps atoms [30,31] and lead to an $n$-dependent detection rate downstream. However, the $\gamma$-ray signal resulting from collisions with the vacuum chamber wall has no discernible dependence on $n$, and the similarity of the TOF spectra for higher $n$ also suggests that there is no significant Ps deflection. Therefore, the most likely loss mechanisms are (1) field ionization upon leaving the excitation region, (2) direct in-flight annihilation of the Rydberg atoms, (3) annihilation following transitions induced by blackbody radiation, and (4) fluorescence and subsequent annihilation from the ground state.

As is evident from Fig. 9(a), complete field ionization of states with $n \geqslant 23$ occurs in the electric fields used in the experiments. There is also some field ionization for lower- $n$ states, which will occur in lower electric fields for the outermost high-field-seeking Stark states [29]. The maximum electric field outside the excitation region is $\sim 900 \mathrm{~V}$ [see Fig. 1(b)]. This is sufficient to ionize some high-field-seeking states with $n=20$ [38], and a small $(\sim 10 \%)$ decrease in the total NaI count rate is observed for $n=20$ compared to $n=18$ (see Fig. 10). However, only the loss of $m=0$ Stark states will significantly affect the mean Ps lifetime (see Fig. 7), resulting in a slightly longer average lifetime. Since all accessible $m$ 

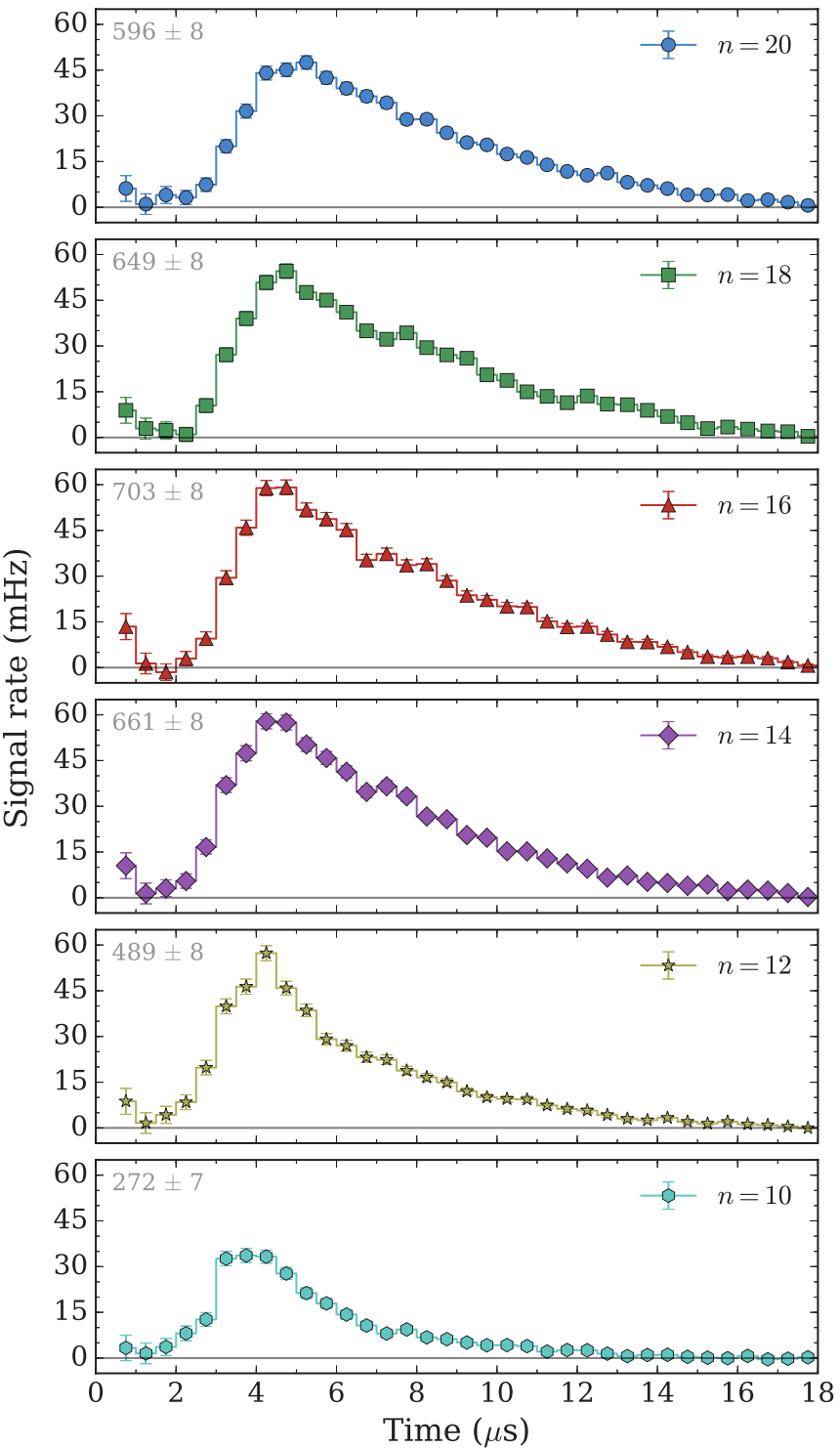

FIG. 10. TOF spectra for Ps atoms in Rydberg states with values of $n=10-20$ measured $1.2 \mathrm{~m}$ from the excitation region using $\mathrm{NaI}$ detectors. The points represent the above-background event rate in each 500-ns time bin. Data were recorded for all states with values of $n$ from 10 to 20 , but only alternate spectra are shown. The total signal rate over the time window $2-18 \mu \mathrm{s}$ is indicated in the top-left corner of each spectrum.

states are expected to be approximately equally populated and the $n=20$ lifetime is already much longer than the flight time, we expect these effects to be negligible in the present measurements.

As previously reported with regard to the photoexcitation of Ps to states with $n=2$, singlet-triplet mixing in the presence of weak magnetic fields can be significantly affected by the addition of electric fields [67]. The increased sensitivity of highly excited Rydberg states to electric fields can further enhance this effect. Although direct annihilation of excited states with $\ell \geqslant 1$ is strongly suppressed, this is not the case for the singlet $s$ states with $\ell=0$. Because the Ps atoms photoexcited in the experiments described here are initially in their triplet ground state, direct singlet-s annihilation in the
Rydberg states can only occur following singlet-triplet mixing induced by the electric and magnetic fields in the experimental apparatus. If this mixing does occur, the maximal singlet- $s$ contribution to any excited state would lead to it possessing $1 /(2 n)$ singlet- $n s$ character. This would result in a lower bound on the average lifetime in the experiments (including both fluorescence and direct annihilation) of 1.7, 8.6, and $27 \mu \mathrm{s}$, at $n=10,15$, and 20, respectively, compared to 2.2, 11, and $35 \mu \mathrm{s}$ with no direct annihilation. There may therefore be some contribution to our measured lifetimes from direct annihilation. However, as these numbers assume complete mixing, we expect that the actual contributions would be significantly less.

The triplet Rydberg states prepared in the experiments can by depopulated by transitions driven by blackbody radiation. In the room-temperature environment in which the experiments were performed, the time scales for $\Delta n=1$ blackbody transitions between $\ell$-mixed Rydberg states of Ps are approximately 20,50, and $70 \mu$ s for initial states with $n=10,15$, and 20, respectively. Such $\Delta n=1$ transitions give the greatest contribution to the rates of blackbody depopulation of the initially prepared states. Although these time scales indicate that blackbody transitions are likely to occur in the experiments, they also suggest that they will not dominate or significantly modify the rates of decay by fluorescence.

If the only significant loss mechanism of Rydberg states is fluorescence and the Rydberg Ps velocity distributions are constant for all values of $n$, it is possible to make a quantitative estimate of the fluorescence lifetimes using the TOF data. To do so it is assumed that the measured distribution for $n=20$ is

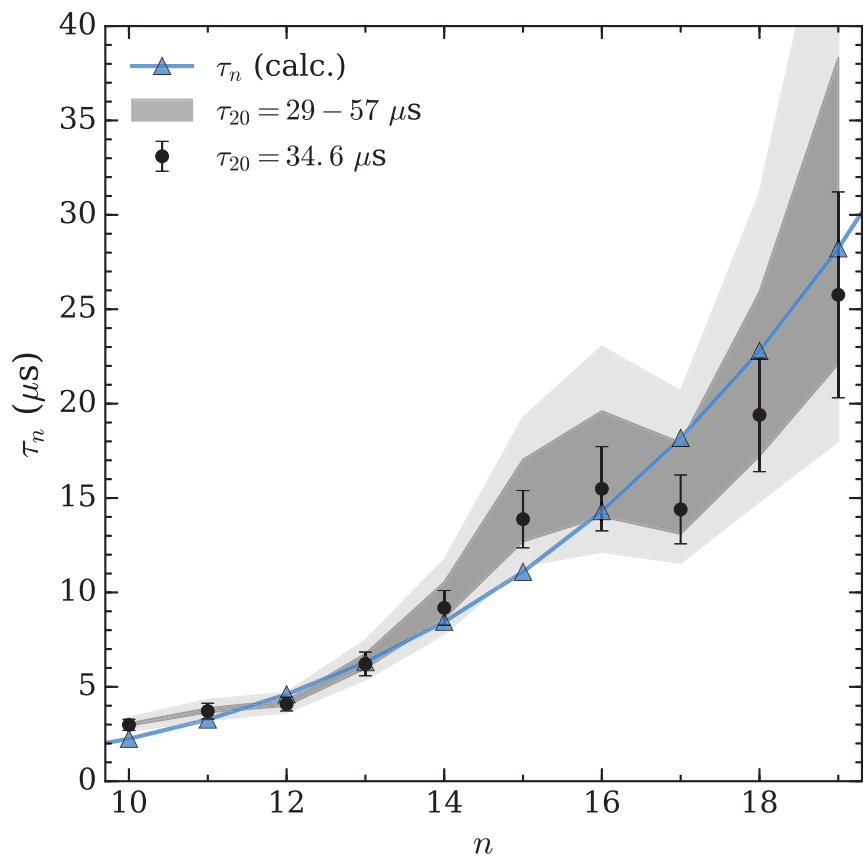

FIG. 11. The overall mean lifetime for all $n$ states measured, obtained using the procedure described in the text. The dark shaded region indicates the range of $\tau$ obtained for different values of $\tau_{20}$ as shown in the legend, with an error indicated by the light shaded region. Also shown are the calculated lifetimes averaged over all $m$ states $\left[\tau_{\mathrm{n}}(\right.$ calc. $\left.)\right]$. 
not subject to significant loss by fluorescence, and the lifetime of this state is $34.6 \mu \mathrm{s}$, the average calculated value for all accessible $m$ states, as shown in Fig. 8. This estimate is based upon the fact that the laser polarization used results in the population of all $m$ states [38] and that the transitions are saturated, as indicated by the data shown in Fig. 9(a). This allows us to determine the underlying TOF distribution with no radiative decay:

$$
f_{\infty}(t)=\frac{1}{\alpha_{n}} \exp \left(\frac{t}{\tau_{n}}\right) f_{n}(t),
$$

where $\alpha_{n}$ is a scaling factor. Using $n=20$ as a reference to obtain $f_{\infty}$, a least-squares minimization routine finds $\tau_{n}$ for each of the other TOF distributions, as shown in Fig. 11. The central shaded region in this figure indicates the range of fluorescence lifetimes that would be obtained if the $n=20$ lifetimes for $m=0,1$, or 2 were used. The fact that the TOF spectra measured using atoms with values of $n \geqslant 14$ are very similar means that the lifetimes obtained for these states are extremely sensitive to the choice of $\tau_{20}$. However, for those states that exhibit significant fluorescence (i.e., those with values of $n \leqslant 13$ ), the lifetimes obtained are very insensitive to the details of the analysis. The measured lifetimes support the expectation that singlet-triplet mixing is not maximal in our experiments and that the contribution of direct Rydberg-state annihilation is not significant.

\section{CONCLUDING REMARKS}

We have reported TOF measurements of Ps atoms in Rydberg states with principal quantum numbers ranging from $n=10-20$. From these data we have determined the fluorescence lifetimes of states with $n=10-19$ using the $n=20$ spectrum as a reference. The measured lifetimes scale with $n^{4}$ and are representative of the mixed- $\ell$ character of hydrogenic Rydberg states photoexcited in the presence of weak electric fields. The lifetimes of Ps states accessible using this methodology are much longer than those of pure- $\ell$ states, and can facilitate experimental schemes requiring very long-lived atoms.

Generating Ps with long lifetimes is an essential step towards a Ps free-fall gravity measurement [68]. This challenging experiment will require atoms that fall for several milliseconds to produce an observable deflection. For the same low $|m|$ states as generated in the present work, this would require Ps atoms excited to states with values of $n \approx 50$. These can already be produced, although in our current experimental arrangement states with $n>20$ are field ionized upon leaving the excitation region. This problem could be eliminated by using a different electrode structure to gradually lower the field or by pulsing the electric fields. Resolving states with $n>30$ would require a laser with a much narrower bandwidth, and the implementation of a Doppler-free excitation scheme (e.g., a single-color two-photon excitation [69]). This would also enable selected excitation of individual $k$ states at higher $n$, and thus produce long-lived Ps atoms with well-defined electric dipole moments, tunable from zero to more than 10,000 D.

\section{ACKNOWLEDGMENTS}

This work was supported by UCL through its Impact Studentship Programme and was funded in part by the ERC (Grant No. CIG 630119) and the EPSRC (Grant No. $\mathrm{EP} / \mathrm{K} 028774 / 1)$. We are grateful to L. Liszkay for providing silica samples.
[1] A. Rich, Recent experimental advances in positronium research, Rev. Mod. Phys. 53, 127 (1981).

[2] D. W. Gidley, H. G. Peng, and R. S. Vallery, Positron annihilation as a method to characterize porous materials, Annu. Rev. Mater. Res. 36, 49 (2006).

[3] S. C. Ellis and J. Bland-Hawthorn, Possibility of observable signatures of leptonium from astrophysical sources, Phys. Rev. D 91, 123004 (2015).

[4] S. J. Brawley, S. Armitage, J. Beale, D. E. Leslie, A. I. Williams, and G. Laricchia, Electron-like scattering of positronium, Science 330, 789 (2010).

[5] T. Yamazaki, T. Namba, S. Asai, and T. Kobayashi, Search for $C P$ Violation in Positronium Decay, Phys. Rev. Lett. 104, 083401 (2010).

[6] R. E. Lingenfelter, J. C. Higdon, and R. E. Rothschild, Is There a Dark Matter Signal in the Galactic Positron Annihilation Radiation? Phys. Rev. Lett. 103, 031301 (2009).

[7] A. Friedland and M. Giannotti, Astrophysical Bounds on Photons Escaping into Extra Dimensions, Phys. Rev. Lett. 100, 031602 (2008).

[8] A. Badertscher, P. Crivelli, W. Fetscher, U. Gendotti, S. N. Gninenko, V. Postoev, A. Rubbia, V. Samoylenko, and D. Sillou, Improved limit on invisible decays of positronium, Phys. Rev. D 75, 032004 (2007).
[9] G. S. Adkins, M. Kim, C. Parsons, and R. N. Fell, Three-PhotonAnnihilation Contributions to Positronium Energies at Order $m \alpha^{7}$, Phys. Rev. Lett. 115, 233401 (2015).

[10] M. Puchalski, A. Czarnecki, and S. G. Karshenboim, Positronium-Ion Decay, Phys. Rev. Lett. 99, 203401 (2007).

[11] S. G. Karshenboim, Precision study of positronium: Testing bound state QED theory, Int. J. Mod. Phys. A 19, 3879 (2004).

[12] M. W. Ritter, P. O. Egan, V. W. Hughes, and K. A. Woodle, Precision determination of the hyperfine-structure interval in the ground state of positronium. V, Phys. Rev. A 30, 1331 (1984).

[13] A. P. Mills, Jr. and G. H. Bearman, New Measurement of the Positronium Hyperfine Interval, Phys. Rev. Lett. 34, 246 (1975).

[14] A. H. Al-Ramadhan and D. W. Gidley, New Precision Measurement of the Decay Rate of Singlet Positronium, Phys. Rev. Lett. 72, 1632 (1994).

[15] R. S. Vallery, P. W. Zitzewitz, and D. W. Gidley, Resolution of the Orthopositronium-Lifetime Puzzle, Phys. Rev. Lett. 90, 203402 (2003).

[16] M. S. Fee, A. P. Mills, Jr., S. Chu, E. D. Shaw, K. Danzmann, R. J. Chichester, and D. M. Zuckerman, Measurement of the Positronium $1^{3} S_{1}-2^{3} S_{1}$ Interval by Continuous-Wave TwoPhoton Excitation, Phys. Rev. Lett. 70, 1397 (1993). 
[17] A. P. Mills, Jr., Measurement of the Decay Rate of the Positronium Negative Ion, Phys. Rev. Lett. 50, 671 (1983).

[18] H. Ceeh, C. Hugenschmidt, K. Schreckenbach, S. A. Gärtner, P. G. Thirolf, F. Fleischer, and D. Schwalm, Precision measurement of the decay rate of the negative positronium ion $\mathrm{Ps}^{-}$, Phys. Rev. A 84, 062508 (2011).

[19] A. I. Alekseev, Two-photon annihilation of positronium in the p-state, Sov. Phys. JETP 7, 826 (1958).

[20] A. I. Alekseev, Three-photon annihilation of positronium in the p-state, Sov. Phys. JETP 9, 1312 (1959).

[21] H. A. Bethe and E. E. Salpeter, Quantum Mechanics of Oneand Two-Electron Atoms (Springer, New York, 1957).

[22] A. C. L. Jones, H. J. Goldman, Q. Zhai, P. Feng, H. W. K. Tom, and A. P. Mills, Jr., Monoenergetic Positronium Emission from Metal-Organic Framework Crystals, Phys. Rev. Lett. 114, 153201 (2015).

[23] A. C. L. Jones, T. H. Hisakado, H. J. Goldman, H. W. K. Tom, A. P. Mills, Jr., and D. B. Cassidy, Doppler-corrected Balmer spectroscopy of Rydberg positronium, Phys. Rev. A 90, 012503 (2014).

[24] S. Chu, A. P. Mills, Jr., and J. L. Hall, Measurement of The Positronium $1^{3} S_{1}-2^{3} S_{1}$ Interval by Doppler-Free Two-Photon Spectroscopy, Phys. Rev. Lett. 52, 1689 (1984).

[25] M. Charlton, Antihydrogen production in collisions of antiprotons with excited states of positronium, Phys. Lett. A 143, 143 (1990).

[26] A. S. Kadyrov, C. M. Rawlins, A. T. Stelbovics, I. Bray, and M. Charlton, Antihydrogen Formation Via Antiproton Scattering with Excited Positronium, Phys. Rev. Lett. 114, 183201 (2015).

[27] A. R. Swann, D. B. Cassidy, A. Deller, and G. F. Gribakin, Formation of positron-atom bound states in collisions between Rydberg Ps and neutral atoms, Phys. Rev. A 93, 052712 (2016).

[28] A. Osterwalder and F. Merkt, Using High Rydberg States As Electric Field Sensors, Phys. Rev. Lett. 82, 1831 (1999).

[29] T. F. Gallagher, Rydberg Atoms (Cambridge University Press, Cambridge, UK, 1994).

[30] T. Breeden and H. Metcalf, Stark Acceleration of Rydberg Atoms in Inhomogeneous Electric Fields, Phys. Rev. Lett. 47, 1726 (1981).

[31] W. H. Wing, Electrostatic Trapping of Neutral Atomic Particles, Phys. Rev. Lett. 45, 631 (1980).

[32] E. Vliegen and F. Merkt, Normal-Incidence Electrostatic Rydberg Atom Mirror, Phys. Rev. Lett. 97, 033002 (2006).

[33] S. D. Hogan and F. Merkt, Demonstration of Three-Dimensional Electrostatic Trapping of State-Selected Rydberg Atoms, Phys. Rev. Lett. 100, 043001 (2008).

[34] S. D. Hogan, P. Allmendinger, H. Saßmannshausen, H. Schmutz, and F. Merkt, Surface-Electrode Rydberg-Stark Decelerator, Phys. Rev. Lett. 108, 063008 (2012).

[35] A. Deller, B. S. Cooper, T. E. Wall, and D. B. Cassidy, Positronium emission from mesoporous silica studied by laserenhanced time-of-flight spectroscopy, New J. Phys. 17, 043059 (2015).

[36] K. P. Ziock, R. H. Howell, F. Magnotta, R. A. Failor, and K. M. Jones, First Observation of Resonant Excitation of High- $n$ States in Positronium, Phys. Rev. Lett. 64, 2366 (1990).

[37] D. B. Cassidy, T. H. Hisakado, H. W. K. Tom, and A. P. Mills, Efficient Production of Rydberg Positronium, Phys. Rev. Lett. 108, 043401 (2012).
[38] T. E. Wall, A. M. Alonso, B. S. Cooper, A. Deller, S. D. Hogan, and D. B. Cassidy, Selective Production of RydbergStark States of Positronium, Phys. Rev. Lett. 114, 173001 (2015).

[39] F. Merkt and R. N. Zare, On the lifetimes of Rydberg states probed by delayed pulsed field ionization, J. Chem. Phys. 101, 3495 (1994).

[40] A. C. L. Jones, H. J. Goldman, H. W. K. Tom, and A. P. Mills, Jr., Polarization dependence of $n=2$ positronium transition rates to Stark-split $n=30$ levels via crossed-beam spectroscopy, J. Phys. B: At., Mol. Opt. Phys. 49, 064006 (2016).

[41] T. F. Gallagher, S. A. Edelstein, and R. M. Hill, Radiative lifetimes of the $S$ and $D$ Rydberg levels of Na, Phys. Rev. A 11, 1504 (1975).

[42] W. P. Spencer, A. G. Vaidyanathan, D. Kleppner, and T. W. Ducas, Measurements of lifetimes of sodium Rydberg states in a cooled environment, Phys. Rev. A 24, 2513 (1981).

[43] A. L. de Oliveira, M. W. Mancini, V. S. Bagnato, and L. G. Marcassa, Measurement of Rydberg-state lifetimes using cold trapped atoms, Phys. Rev. A 65, 031401 (2002).

[44] M. Mack, J. Grimmel, F. Karlewski, L. Sárkány, H. Hattermann, and J. Fortágh, All-optical measurement of Rydberg-state lifetimes, Phys. Rev. A 92, 012517 (2015).

[45] E. Vliegen, H. J. Wörner, T. P. Softley, and F. Merkt, Nonhydrogenic Effects in the Deceleration of Rydberg Atoms in Inhomogeneous Electric Fields, Phys. Rev. Lett. 92, 033005 (2004).

[46] S. D. Hogan, Rydberg-Stark deceleration of atoms and molecules, EPJ Tech. Instrum. 3, 1 (2016).

[47] Ch. Seiler, S. D. Hogan, H. Schmutz, J. A. Agner, and F. Merkt, Collisional and Radiative Processes in Adiabatic Deceleration, Deflection, and Off-Axis Trapping of a Rydberg Atom Beam, Phys. Rev. Lett. 106, 073003 (2011).

[48] Ch. Seiler, S. D. Hogan, and F. Merkt, Dynamical processes in Rydberg-Stark deceleration and trapping of atoms and molecules, Chimia 66, 208 (2012).

[49] A. P. Mills, Jr., Thermal activation measurement of positron binding energies at surfaces, Solid State Commun. 31, 623 (1979).

[50] S. Berko and H. N. Pendleton, Positronium, Annu. Rev. Nucl. Part. Sci. 30, 543 (1980).

[51] P. J. Schultz and K. G. Lynn, Interaction of positron beams with surfaces, thin films, and interfaces, Rev. Mod. Phys. 60, 701 (1988).

[52] B. S. Cooper, A. M. Alonso, A. Deller, T. E. Wall, and D. B. Cassidy, A trap-based pulsed positron beam optimized for positronium laser spectroscopy, Rev. Sci. Instrum. 86, 103101 (2015).

[53] A. P. Mills, Jr. and E. M. Gullikson, Solid neon moderator for producing slow positrons, Appl. Phys. Lett. 49, 1121 (1986).

[54] J. R. Danielson, D. H. E. Dubin, R. G. Greaves, and C. M. Surko, Plasma and trap-based techniques for science with positrons, Rev. Mod. Phys. 87, 247 (2015).

[55] A. P. Mills, Jr., Time bunching of slow positrons for annihilation lifetime and pulsed laser photon absorption experiments, Appl. Phys. 22, 273 (1980).

[56] L. Liszkay, M.-F. Barthe, C. Corbel, P. Crivelli, P. Desgardin, M. Etienne, T. Ohdaira, P. Perez, R. Suzuki, V. Valtchev, and 
A. Walcarius, Orthopositronium annihilation and emission in mesostructured thin silica and silicalite-1 films, Appl. Surf. Sci. 255, 187 (2008).

[57] L. Liszkay, F. Guillemot, C. Corbel, J.-P. Boilot, T. Gacoin, E. Barthel, P. Pérez, M.-F. Barthe, P. Desgardin, P. Crivelli, U. Gendotti, and A. Rubbia, Positron annihilation in LaTeX-templated macroporous silica films: Pore size and ortho-positronium escape, New J. Phys. 14, 065009 (2012).

[58] W. Yantasee, Y. Lin, X. Li, G. E. Fryxell, T. S. Zemanian, and V. V. Viswanathan, Nanoengineered electrochemical sensor based on mesoporous silica thin-film functionalized with thiolterminated monolayer, Analyst 128, 899 (2003).

[59] D. B. Cassidy, P. Crivelli, T. H. Hisakado, L. Liszkay, V. E. Meligne, P. Perez, H. W. K. Tom, and A. P. Mills, Jr., Positronium cooling in porous silica measured via Doppler spectroscopy, Phys. Rev. A 81, 012715 (2010).

[60] P. Crivelli, U. Gendotti, A. Rubbia, L. Liszkay, P. Perez, and C. Corbel, Measurement of the orthopositronium confinement energy in mesoporous thin films, Phys. Rev. A 81, 052703 (2010).

[61] D. B. Cassidy, S. H. M. Deng, H. K. M. Tanaka, and A. P. Mills, Jr., Single shot positron annihilation lifetime spectroscopy, Appl. Phys. Lett. 88, 194105 (2006).
[62] D. B. Cassidy and A. P. Mills, Jr., A fast detector for singleshot positron annihilation lifetime spectroscopy, Nucl. Instrum. Methods Phys. Res., Sect. A 580, 1338 (2007).

[63] John R. Hiskes, C. Bruce Tarter, and D. A. Moody, Stark lifetimes for the hydrogen atom, Phys. Rev. 133, A424 (1964).

[64] Y. Nagashima, Y. Morinaka, T. Kurihara, Y. Nagai, T. Hyodo, T. Shidara, and K. Nakahara, Origins of positronium emitted from $\mathrm{SiO}_{2}$, Phys. Rev. B 58, 12676 (1998).

[65] C. Tianbao, X. Min, and Z. Xiangtao, Effect of the energy loss process on the annihilation of orthopositronium in silica aerogel, Phys. Lett. A 126, 189 (1987).

[66] J. Algers, P. Sperr, W. Egger, G. Kögel, and F. H. J. Maurer, Median implantation depth and implantation profile of 3-18 keV positrons in amorphous polymers, Phys. Rev. B 67, 125404 (2003).

[67] A. M. Alonso, B. S. Cooper, A. Deller, S. D. Hogan, and D. B. Cassidy, Controlling Positronium Annihilation with Electric Fields, Phys. Rev. Lett. 115, 183401 (2015).

[68] A. P. Mills, Jr. and M. Leventhal, Can we measure the gravitational free fall of cold Rydberg state positronium? Nucl. Instrum. Meth. 192, 102 (2002).

[69] T. E. Wall, D. B. Cassidy, and S. D. Hogan, Single-color twophoton spectroscopy of Rydberg states in electric fields, Phys. Rev. A 90, 053430 (2014). 